\title{
Does SARS-CoV-2 infection cause chronic neurological complications?
}

\author{
Erin R. Hascup $\mathbb{D}$ - Kevin N. Hascup $\mathbb{D}$
}

Received: 21 April 2020 / Accepted: 19 May 2020 / Published online: 25 May 2020

(C) American Aging Association 2020

\begin{abstract}
The current pandemic caused by severe acute respiratory syndrome coronavirus (SARSCoV)-2 has created an unparalleled health crisis. Besides the acute respiratory infection, $\mathrm{CoVs}$ are neuroinvasive causing additional inflammation and neurodegeneration. This is likely also true of SARSCoV-2 given reports of neurological manifestations in coronavirus disease 2019 (COVID-19) positive patients. Older adults $>65$ years of age constitute a high-risk group prone to severe infection and death. Despite the higher mortality rate, a majority of cases are expected to recover and survive from this viral outbreak. But, the long-term consequences of SARS-CoV-2 neuroinfection are unknown. We discuss these potential chronic changes to the central nervous system (CNS) in relation to accelerated brain aging and age-related neurodegenerative disorders.
\end{abstract}

Keywords COVID-19. Cellular senescence . Alzheimer's disease $\cdot$ Neurotropism $\cdot$ Aging . Neurodegeneration

\section{E. R. Hascup • K. N. Hascup $(\bowtie)$}

Department of Neurology, Center for Alzheimer's Disease and Related Disorders Department of Pharmacology, Neurosciences Institute, P.O. Box 19628, Springfield, IL 62794-9628, USA e-mail: khascup49@siumed.edu

K. N. Hascup

Department of Medical Microbiology, Immunology, and Cell Biology, Southern Illinois University School of Medicine, Springfield, IL, USA

\begin{abstract}
Abbreviations
ACE2 Angiotensin converting enzyme 2

BBB Blood-brain barrier

BDNF Brain-derived neurotropic factor

CNS Central nervous system

COVID-19 Coronavirus disease 2019

OPCs Oligodendrocyte progenitor cells

SASP Senescence-associated secretory phenotype

SARS-CoV Severe acute respiratory syndrome coronavirus
\end{abstract}

Severe acute respiratory syndrome coronavirus SARS$\mathrm{CoV}-2$ belongs to the $\beta-\mathrm{CoV}$ family and shares a highly homologous sequence with SARS-CoV. Host cell infection is mediated by binding of the spike glycoprotein to the angiotensin-converting enzyme 2 (ACE2) that is distributed throughout the body including respiratory tract epithelia, lung parenchyma, cardiomyocytes, vascular endothelia, gastrointestinal tract, and the central nervous system (CNS). The majority of cases involve fever, dry cough, and lethargy; however, older adults are more susceptible to severe infection involving pneumonia, dyspnea, and acute respiratory distress syndrome resulting in a higher mortality rate. A natural decline in ACE2 expression and the subsequent pro-inflammatory profile with aging, "inflamm-aging", may explain the increased severity and comorbid diabetic and hypertensive complications observed in older adults (Peña Silva et al. 2012; AlGhatrif et al. 2020). While a majority of 
cases are expected to recover and survive from this viral outbreak, the long-term sequelae on aging and neurodegenerative disorders are currently unknown. We discuss potential long-term implications of SARS-CoV-2 infection in relation to accelerated brain aging, neurovascular coupling, and age-related neurodegenerative disorders.

CoVs are neurotrophic and neuroinvasive pathogens (Desforges et al. 2014), and initial reports indicate neurological manifestations are correlated with the severity of SARS-CoV-2 infection, which include loss of consciousness, smell, taste, vision, nerve pain, ataxia, and seizures (Mao et al. 2020). Neurotropic pathogens access the CNS through multiple routes including retrograde axonal transport along olfactory and enteric neurons or infection of blood lymphocytes passing through a disrupted blood-brain barrier (BBB). A gradual deterioration of the $\mathrm{BBB}$ is observed in normal aging (Montagne et al. 2015) that may indicate increased neurotrophic potential in older adults. Once present in the CNS, CoVs can cause demyelination, neurodegeneration, and cellular senescence, all of which accelerate brain aging and potentially exacerbate underlying neurodegenerative pathology.

\section{Neurocardiac Axis}

Numerous cortical and subcortical structures are involved in a complex communication between the cardiovascular and nervous system to maintain healthy cardiac contraction controlled by both sympathetic and parasympathetic outflow. Brain-derived neurotropic factor (BDNF) represents additional mechanisms linking this neurocardiac axis. Besides its role in neurogenesis, axonal sprouting, and memory consolidation, BDNF is also responsible for vasculogenesis and survival of both cardiomyocytes and endothelial cells. Decreased BDNF, in conjunction with a subsequent rise in oxidative stress, has been observed in normal aging and is thought to precipitate the onset of dementia (Diniz et al. 2014), as well as progression of cardiovascular disease. SARS-CoV-2 can infect cardiomyocytes expressing ACE2 receptors causing elevated reactive oxygen species resulting in cellular damage. The decreased BDNF expression in aged individuals perturbs repair mechanisms making aged populations more susceptible to acute cardiac injury. The subsequent hypoperfusion limits the supply of energy substrates to the CNS potentially decreasing BDNF production. This feed-forward mechanism makes the CNS more susceptible to the cellular damage instigated by the acute rise in proinflammatory cytokines and reactive oxygen species. The decreased BDNF expression levels coupled with cellular damage may initiate or exacerbate cognitive decline.

\section{Neurovascular coupling}

Neurons, astrocytes, vascular smooth muscle cells, pericytes, and endothelial cells work in concert to form the $\mathrm{BBB}$ that regulates regional cerebral blood flow in response to altered neuronal activity. Transcytosis allows essential macromolecules into the CNS to maintain neurovascular coupling, hemodynamic responses to support energy demands during neuronal activity. This membrane also maintains the CNS extracellular fluid while preventing neurotoxic compounds and microbial agents from entering the CNS through the blood stream. Aging is associated with a decline in cerebral blood flow and neurovascular coupling in limbic and associate cortices, which has become a hallmark sign of vascular cognitive impairment in older adults (Lipecz et al. 2019). SARS-CoV-2 can infect endothelial cells expressing ACE2 potentially leading to further deterioration of this integral architecture. The resulting hypoperfusion would restrict energy substrates essential for maintaining neuronal networks thereby accelerating cognitive decline in the elderly. Furthermore, disruptions of the neurovascular unit pose a potential viral entry pathway into the CNS causing localized inflammatory and immune responses that initiate neurodegenerative processes.

\section{Demyelination}

The mRNA of several different strains of human $\mathrm{CoV}$ have been observed in postmortem brain tissue of multiple sclerosis (MS) patients (Desforges et al. 2014). Experimentally, murine $\mathrm{CoV}$ has shown chronic viral persistence in oligodendrocytes leading to immunemediated demyelination (Hosking and Lane 2010). Diminished myelin renewal occurs during normal aging in mammals that may be increased upon SARS-CoV-2 infection but delayed until recovery of acute infection. Initially, delayed neurological sequelae resembling MS (ataxia and peripheral neuropathy) may be observed 
post-infection, while damage to limbic and cortical regions could cause retrograde and anterograde amnesia. The duration of these symptoms post-infection is dependent upon oligodendrocyte progenitor cells (OPCs) differentiation and subsequent remyelination of affected nerve tissue. Since ACE2 is found on OPCs (Chen et al. 2020), SARS-CoV-2 infection may adversely affect oligodendrocyte differentiation causing these demyelinating conditions to become chronic or even slowly deteriorate over time.

\section{Neurodegeneration}

Although physiological brain aging is characterized by neuroinflammation, synaptic pruning, and neuronal loss that underlies an age-dependent decline in sensory, cognitive, and motor performance, SARS-CoV-2 infection may accelerate this process. As a result of ACE2 downregulation, SARS-CoV-2 infection in older adults induces aggressive secretion of pro-inflammatory cytokines. Once present in the blood stream, these cytokines can be actively transported across the BBB initiating a neuroinflammatory response from astrocytes and microglial. Pro-inflammatory cytokines increases oxidative stress that damages cellular membranes and downregulates surface expression of excitatory amino acid transporters that are necessary for terminating glutamatergic signaling. The resulting elevated glutamate levels can lead to an excitotoxic environment precipitating the neuronal loss and initiating a vicious feedforward cycle that causes further damage to the surrounding parenchyma. Furthermore, the presence of ACE2 receptors on GABAergic and glutamatergic neurons (Chen et al. 2020) indicates that CoVs have the potential to enter these neurons, but the consequences of their incorporation are largely unknown. Viral entry may create a cytotoxic insult and initiate apoptotic pathways or create an excitatory-inhibitory imbalance, which is already postulated to play a role in several neurodegenerative diseases including Alzheimer's and Parkinson's disease. Synaptic signaling may also provide a means of $\mathrm{CoV}$ spread along projections through a prion-like mechanism to CNS regions without ACE2 expression. A slow infiltration throughout the CNS may precipitate underlying pathologies associated with age-related neurodegenerative disorders months or years after acute viral infection.

\section{Cellular senescence}

Cellular senescence is characterized by a permanently arrested cell cycle that is no longer responsive to differentiation and apoptotic signaling processes. Senescent cells naturally accumulate with age throughout the body and have recently been implicated in age-related neurodegenerative disorders including Alzheimer's disease. Senescent cells continue to be metabolically active and undergo senescence-associated secretory phenotype (SASP) causing secretion of pro-inflammatory cytokines and chemokines, not unlike what is observed in SARS-CoV-2 infected patients. This may indicate SARS-CoV-2 infection induces a senescent phenotype particularly in the lower respiratory tract. This phenomenon has been observed experimentally with other viruses as a potential host response to limit viral replication while signaling an innate and adaptive immunological response (Baz-Martínez et al. 2016).

The neuroinvasive potential of SARS-CoV-2 may result in senescence of several different CNS cell types. Senescent oligodendrocytes or OPCs would no longer be able to remyelinate axons thereby increasing the refractory period for action potential propagation. Astrocytic endfeet are integral members of the BBB and are important for distribution of macromolecules into the CNS. Accordingly, senescent astroglia may compromise the BBB integrity while limiting the distribution of substrates important for maintaining the metabolic demands of neuronal networks. While neurons exist in a post-mitotic state, neural stem cells can differentiate into neurons that integrate into the granule layer. If neural stem cells underwent senescence after viral infection, they would no longer be able to undergo neurogenesis in a brain region critical to memory consolidation. Finally, the SASP and peripheral upregulation of proinflammatory cytokines could synergistically create a feed-forward cycle causing neuroinflammation to the surrounding parenchyma further accelerating aging and age-related neurodegenerative processes.

\section{Interventional strategies}

Currently, development of antiviral medications to slow or modify disease progression is rapidly progressing; however, large-scale dissemination is years away. In the meantime, neuro- and cardioprotective strategies are needed that can reduce both the risk and severity 
of SARS-CoV-2 infection while also improving recovery in COVID-19 patients. The heightened severity of infection in patients with pre-existing vascular disease suggests lifestyle modifications known to extend lifespan and healthspan may provide therapeutic benefit. Minimizing caloric intake either by fasting or meal timing is known to postpone disease onset and delay aging in almost every mammalian species. However, implementation and adherence to this dietary regimen is difficult. Because of this, anti-aging therapeutics may provide additional sources of protection or improve recovery. For example, sirtuins are a group of proteins that regulate inflammation and mitochondrial energy homeostasis through $\mathrm{NAD}^{+}$-dependent deacetylation. Drugs targeting these proteins promote neurovascular rejuvenation and cardioprotection (Kiss et al. 2020) and may be beneficial in COVID-19 patients to speed recovery. Finally, a hallmark of aging is the accumulation of senescent cells throughout the body. Senotherapeutics such as Quercetin and Fisetin have been shown experimentally to improve health and lifespan. These compounds may prove beneficial postinfection to both cardiac and CNS cells thereby mitigating the effects of viral infection.

\section{Concluding remarks}

BBB deterioration in older adults leaves them more susceptible to neuroinvasion during SARS-CoV-2 infection. After the acute recovery phase, the long-term consequences on accelerated aging and age-related neurodegenerative disorders are unknown. Viral aggravation of underlying neuropathologies has the potential to hasten the onset of or further deteriorate motor and cognitive deficits. Prior to this pandemic, the number of Alzheimer's and Parkinson's disease patients was rapidly rising due to our aging demographic and a lack of disease modifying therapies. When this viral outbreak is managed, our healthcare system could face an increased volume of patients dealing with these and their associated comorbid neurological issues. As such, long-term neurological follow-up in older adults may be needed after severe SARSCoV-2 infection.

\footnotetext{
Author contributions ERH and $\mathrm{KNH}$ wrote and revised the manuscript.
}

Funding information This work was supported by the National Institutes of Health (NIA R01AG057767 and NIA R01AG061937), from the SIU Foundation at the School of Medicine (Harriss and Fannie Belle Roe Malan Research Endowment and the Illinois Health Improvement Association Research Endowment), the Center for Alzheimer's Disease and Related Disorders, and the Kenneth Stark Endowment.

\section{Compliance with ethical standards}

Conflict of interest The authors declare that they have no competing interests.

\section{References}

AlGhatrif M, Cingolani O, Lakatta EG. The dilemma of coronavirus disease 2019, aging, and cardiovascular disease. JAMA Cardiol. 2020. https://doi.org/10.1001 /jamacardio.2020.1329.

Baz-Martínez M, Da Silva-Álvarez S, Rodríguez E, et al. Cell senescence is an antiviral defense mechanism. Sci Rep. 2016;6. https://doi.org/10.1038/srep37007.

Chen R, Wang K, Yu J, et al (2020) The spatial and cell-type distribution of SARS-CoV-2 receptor ACE2 in human and mouse brain. bioRxiv 2020.04.07.030650. https://doi. org/10.1101/2020.04.07.030650.

Desforges M, Le Coupanec A, Stodola JK, et al. Human coronaviruses: viral and cellular factors involved in neuroinvasiveness and neuropathogenesis. Virus Res. 2014;194:145-58.

Diniz BS, Reynolds CF, Begley A, et al. Brain-derived neurotrophic factor levels in late-life depression and comorbid mild cognitive impairment: a longitudinal study. J Psychiatr Res. 2014;49:96-101. https://doi. org/10.1016/j.jpsychires.2013.11.004.

Hosking MP, Lane TE. The pathogenesis of murine coronavirus infection of the central nervous system. Crit Rev Immunol. 2010;30:119-30.

Kiss T, Nyúl-Tóth Á, Balasubramanian P, Tarantini S, Ahire C, Yabluchanskiy A, et al. Nicotinamide mononucleotide (NMN) supplementation promotes neurovascular rejuvenation in aged mice: transcriptional footprint of SIRT1 activation, mitochondrial protection, anti-inflammatory, and antiapoptotic effects. GeroScience. 2020;42:527-46. https://doi. org/10.1007/s11357-020-00165-5.

Lipecz A, Csipo T, Tarantini S, Hand RA, Ngo BTN, Conley S, et al. Age-related impairment of neurovascular coupling responses: a dynamic vessel analysis (DVA)-based approach to measure decreased flicker light stimulus-induced retinal arteriolar dilation in healthy older adults. GeroScience. 2019;41: 341-9. https://doi.org/10.1007/s11357-019-00078-y.

Mao L, Jin H, Wang M, Hu Y., Chen S., He Q., Chang J., Hong C., Zhou Y., Wang D., Miao X., Li Y., Hu B. (2020) Neurologic manifestations of hospitalized patients with coronavirus disease 2019 in Wuhan, China JAMA Neurol https://doi.org/10.1001/jamaneurol.2020.1127. 
Montagne A, Barnes SR, Sweeney MD, Halliday MR, Sagare AP, Zhao Z, et al. Blood-brain barrier breakdown in the aging human hippocampus. Neuron. 2015;85:296-302. https://doi. org/10.1016/j.neuron.2014.12.032.

Peña Silva RA, Chu Y, Miller JD, et al. Impact of ACE2 deficiency and oxidative stress on cerebrovascular function with aging. Stroke. 2012;43:3358-63. https://doi.org/10.1161 /STROKEAHA.112.667063.

Publisher's note Springer Nature remains neutral with regard to jurisdictional claims in published maps and institutional affiliations. 